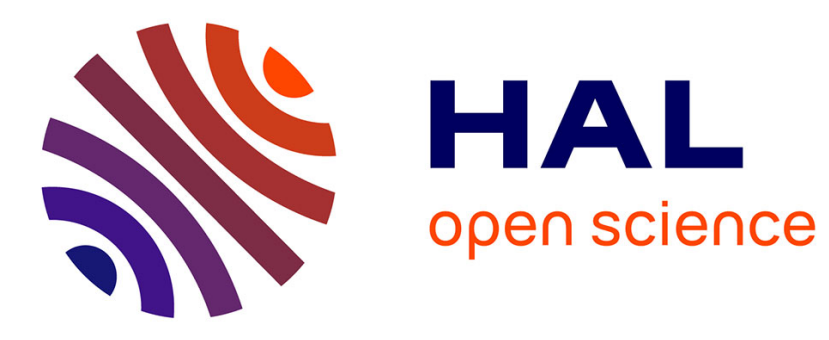

\title{
Hyperelasticity of Soft Tissues and Related Inverse Problems
}

Stéphane Avril

\section{To cite this version:}

Stéphane Avril. Hyperelasticity of Soft Tissues and Related Inverse Problems. Stéphane Avril, Sam Evans Material Parameter Identification and Inverse Problems in Soft Tissue Biomechanics, 573, Springer, pp.37 - 66, 2016, CISM International Centre for Mechanical Sciences, 978-3-319-45070-4. 10.1007/978-3-319-45071-1_2 . hal-01380428

\section{HAL Id: hal-01380428 https://hal.science/hal-01380428}

Submitted on 17 Oct 2016

HAL is a multi-disciplinary open access archive for the deposit and dissemination of scientific research documents, whether they are published or not. The documents may come from teaching and research institutions in France or abroad, or from public or private research centers.
L'archive ouverte pluridisciplinaire $\mathbf{H A L}$, est destinée au dépôt et à la diffusion de documents scientifiques de niveau recherche, publiés ou non, émanant des établissements d'enseignement et de recherche français ou étrangers, des laboratoires publics ou privés. 


\title{
Hyperelasticity of soft tissues and related inverse problems
}

\author{
Stéphane Avril \\ Inserm U1059, Mines Saint-Étienne, University of Lyon, Saint-Étienne, France
}

\begin{abstract}
In this chapter, we are interested in the constitutive equations used to model macroscopically the mechanical function of soft tissues. After reviewing some basics about nonlinear finite-strain constitutive relations, we present recent developments of experimental biomechanics and inverse methods aimed at quantifying constitutive parameters of soft tissues. A focus is given to in vitro characterization of hyperelastic parameters based on full-field data that can be collected with digital image correlation systems during the experimental tests. The specific use of these data for membranelike tissues is first illustrated through the example of bulge inflation tests carried out onto pieces of aortic aneurysms. Then an inverse method, based on the principle of virtual power, is introduced to estimate regional variations of material parameters for more general applications.
\end{abstract}




\section{Introduction}

A better understanding of many issues of human health, disease, injury, and their treatment thereof necessitates a detailed quantification of how biological cells, tissues, and organs respond to applied loads. Thus, experimental and computational mechanics can, and must, play a fundamental role in cell biology, physiology, pathophysiology, and clinical intervention. The goal of this chapter is to discuss some of the recent developments of experimental biomechanics based on the use of digital image correlation and inverse methods for quantifying the finite-strain behaviour of biological soft tissues in terms of nonlinear constitutive relations. After a brief review of these constitutive relations, two recent developments of the author's experience are presented to illustrate the potential of digital image correlation and inverse methods in experimental biomechanics of soft tissues.

\section{Basic constitutive equations of soft tissues}

This section presents the theoretical background for understanding the foundations of constitutive models in soft tissues. This background is essential for the following sections and for the other chapters of this book. The presentation of this background follows the approach of a seminal paper from Millard F Beatty [1].

\subsection{Kinematics of finite deformation}

Since the early 1940's there has been enormous progress in the development of a theory of elastic materials subjected to large deformations. Significant theoretical results, many confirmed by experiments, have projected considerable light on the physical behaviour of rubberlike materials such as synthetic elastomers, polymers and biological tissue, in addition to natural rubber. The mathematical theory of elasticity of materials subjected to large deformations is inherently nonlinear. The theory of elasticity of materials for which there exists an elastic potential energy function is known as hyperelasticity. Before presenting the constitutive equations for a hyperelastic solid, we begin with a sketch of the principal kinematical relations used to describe the finite deformation of a continuum and with the Cauchy stress principle and equations of kinetics. A body $B=\left\{P_{k}\right\}$ is a set of material points $P_{k}$ called particles. A reference frame is a set $\Upsilon=\{O, \mathbf{e}\}$ consisting of an origin point $O$ and an orthonormal vector basis e. The motion of a particle $P$ relative to $\Upsilon$ is described by the time locus of its position vector $\mathbf{x}(P, t)$ relative to $\Upsilon$. This locus is the trajectory or path of $P$ in $\Upsilon$. A typical particle $P$ may be identified by its position vector 
$\mathbf{X}(P)$ in $\Upsilon$ at some reference time $t_{0}$. The domain $\kappa_{0}$ of $\mathbf{X}$, the region in Euclidian space occupied by $B$ at time $t_{0}$ is called a reference configuration of $B$. Then, relative to $\Upsilon$ the motion of a particle $P$ from $\kappa_{0}$ is described by the vector function

$$
\mathbf{x}=\chi(\mathbf{X}, t)
$$

The domain $\kappa$ of $\mathbf{x}$, the region in Euclidian space occupied by $B$ at time $t_{0}$ is called a current configuration of $B$. Hence, $\mathbf{x}$ denotes the place at time $t$ in the current configuration $\kappa$ which is occupied by the particle $P$ whose place was $X$ in the reference configuration of $B$. The velocity and acceleration of a particle $P$ relative to $\Upsilon$ are define by:

$$
\begin{gathered}
\mathbf{v}(\mathbf{X}, t)=\dot{\mathbf{x}}(\mathbf{X}, t) \\
\gamma(\mathbf{X}, t)=\dot{\mathbf{v}}(\mathbf{X}, t)=\ddot{\mathbf{x}}(\mathbf{X}, t)
\end{gathered}
$$

We shall assume henceforward that the nody is a contiguous collection of particles, we call this body a continuum. It is assumed that $\chi$ is a smooth one-to-one map of every material point of $\kappa_{0} \rightarrow \kappa$ with:

$$
J=\operatorname{det} \mathbf{F}>0
$$

in which

$$
\mathbf{F}=\partial \mathbf{x} / \partial \mathbf{X}=\mathbf{G r a d} \mathbf{x}
$$

is called the deformation gradient. This tensor transforms the tangent element $\mathrm{d} \mathbf{X}$ of a material line $\mathfrak{L}_{0}$ in $\kappa_{0}$ into the tangent element $\mathrm{d} \mathbf{x}$ of its deformed image line $\mathfrak{L}$ in $\kappa$. Hence,

$$
\mathrm{d} \mathbf{x}=\mathbf{F} \mathrm{d} \mathbf{X}
$$

Let $\|\mathrm{d} \mathbf{x}\|=\mathrm{d} l$ and $\|\mathrm{d} \mathbf{X}\|=\mathrm{d} L$, where $l$ and $L$ are the arc length parameters for $\mathfrak{L}$ and $\mathfrak{L}_{0}$ respectively. Then Eq. 0.6 may be written:

$$
\lambda \mathbf{e}=\mathbf{F E}
$$

in which $\mathbf{e}=\mathrm{d} \mathbf{x} / \mathrm{d} l$ and $\mathbf{E}=\mathrm{d} \mathbf{X} / \mathrm{d} L$ are unit vectors tangent to $\mathfrak{L}$ and $\mathfrak{L}_{0}$ at $\mathbf{x}$ and $\mathbf{X}$ as shown in Figure and

$$
\lambda=\mathrm{d} l / \mathrm{d} L
$$

is named the stretch, the ratio of the current length $\mathrm{d} s$ to the reference length $\mathrm{d} S$ of the material element. These lengths are commonly called the deformed and undeformed lengths, respectively. However it is not essential that the reference configuration be an undistorted reference configuration, 
nor one that the body need actually occupy at any time during its motion. It is seen that Eq. 0.7 expresses the physical result that $\mathbf{F}$ rotates $\mathbf{E}$ into the direction $\mathbf{e}$ and stretches it by an amount $0<\lambda<\infty$. This is essentially the substance of the more general and physically useful polar decomposition theorem of linear algebra applied pointwise to the nonsingular tensor $\mathbf{F}$ :

$$
\mathbf{F}=\mathbf{R U}=\mathbf{V R}
$$

The proper orthogonal tensor $\mathbf{R}$ characterizes the local rigid body rotation of a material element. The positive symmetric tensors $\mathbf{U}$ and $\mathbf{V}$ describe the local deformation of the element. They are called the right and the left stretch tensors, respectively. The decomposition of the deformation gradient $\mathbf{F}$ into a pure stretch $\mathbf{U}$ at $\mathbf{X}$ followed by a rigid body rotation $\mathbf{R}$, or by the same rigid body rotation followed by a pure stretch $\mathbf{V}$ at $\mathbf{x}$ is unique. Because $\mathbf{U}$ and $\mathbf{V}$ usually are tedious to compute, it is customary to use their squares:

$$
\mathbf{C}=\mathbf{F}^{T} \mathbf{F}=\mathbf{U}^{2} \text { and } \mathbf{B}=\mathbf{F F}^{T}=\mathbf{V}^{2}
$$

The corresponding positive symmetric tensors are respectively known as the right and the left Cauchy-Green deformation tensors. It follows that $\mathbf{U}$ and $\mathbf{V}(\mathbf{C}$ and $\mathbf{B})$ have the same principal values $\lambda_{k}\left(\lambda_{k}^{2}\right)$ and respective principal directions $\boldsymbol{\mu}$ and $\boldsymbol{\nu}$ are related by the rotation $\mathbf{R}$ :

$$
\boldsymbol{\nu}=\mathbf{R} \cdot \boldsymbol{\mu}
$$

The $\lambda_{k}$ are the stretches of the three principal material lines, they are called the principal stretches.

Formulae relating the respective material surface area and volume elements $\mathrm{d} a$ and $\mathrm{d} v$ in $\kappa$ to their respective reference images $\mathrm{d} A$ and $\mathrm{d} V$ in $\kappa_{0}$ may be easily derived by application of:

$$
\mathbf{n d} a=J \mathbf{F}^{-T} \cdot \mathbf{N d} A \text { and } \mathrm{d} v=J \mathrm{~d} V
$$

where $\mathbf{n}$ is the exterior unit normal vector to $\partial \mathscr{P}$ in $\kappa$ and $\mathbf{N}$ is the exterior unit normal vector to $\partial \mathscr{P}$ in $\kappa_{R}$.

The previous relation shows that $\operatorname{det} \mathbf{F}$ is the ratio of the current (deformed) volume to the reference (undeformed) volume of a material element. Therefore the deformation is isochoric if $J=1$. It is evident on physical grounds that $0<\operatorname{det} \mathbf{F}<\infty$. The material time rate of the deformation of a continuum is described by the velocity gradient tensor $\mathbf{L}$ :

$$
\mathbf{L}=\operatorname{Grad} \dot{\mathbf{x}}=\dot{\mathbf{F}} \mathbf{F}^{-1}
$$

The symmetric part $\mathbf{D}$ and antisymmetric part $\mathbf{W}$ of $\mathbf{L}$ are the stretching and spin tensors respectively. 


\subsection{The Cauchy stress principle and the equations of motion}

The forces that act on any part $\mathscr{P} \subset \mathscr{B}$ of a continuum $\mathscr{B}$ are of two kinds: a distribution of contact force $\mathbf{t}_{\mathbf{n}}$ per unit area of the boundary $\partial \mathscr{P}$ of $\mathscr{P}$ in $\kappa$, and a distribution of body force $\mathbf{b}$ per unit volume of $\mathscr{P}$ in $\kappa$. The total force $\mathscr{F}(\mathscr{P}, t)$ and the total torque $\mathscr{T}(\mathscr{P}, t)$ acting on the part $\mathscr{P}$ are related to the momentum and the moment of momentum of the material points of $\mathscr{B}$ in an inertial frame $\Phi$ in accordance with Euler's laws of motion:

$$
\begin{gathered}
\mathscr{F}(\mathscr{P}, t)=\int_{\partial \mathscr{P}} \mathbf{t}_{\mathbf{n}} \mathrm{d} a+\int_{\mathscr{P}} \mathbf{b} \mathrm{d} v=\frac{\mathrm{d}}{\mathrm{d} t} \int_{\mathscr{P}} \mathbf{v} \mathrm{d} m \\
\mathscr{T}(\mathscr{P}, t)=\int_{\partial \mathscr{P}} \mathbf{x} \times \mathbf{t}_{\mathbf{n}} \mathrm{d} a+\int_{\mathscr{P}} \mathbf{x} \times \mathbf{b} \mathrm{d} v=\frac{\mathrm{d}}{\mathrm{d} t} \int_{\mathscr{P}} \mathbf{x} \times \mathbf{v ~} \mathrm{d} m
\end{gathered}
$$

The moments in Eq. 0.15 are to be computed with respect to the origin in $\Phi$. Note that $\mathrm{d} m=\rho \mathrm{d} v$ is the material element of mass with density $\rho$ per unit volume in $\kappa$.

The principle of balance of mass requires also that $\mathrm{d} m=\rho_{R} \mathrm{~d} V$ where $\rho_{R}$ is the density of mass per unit volume $V$ in $\kappa_{R}$. Therefore one finds that the respective mass densities are related by the local equation of continuity:

$$
\rho_{R}=J \rho
$$

Application of the first law of Euler to an arbitrary tetrahedral element leads to Cauchy's stress principle:

$$
\mathbf{t}_{\mathbf{n}}=\boldsymbol{\sigma} \cdot \mathbf{n}
$$

Hence the traction or stress vector $\mathbf{t}_{\mathbf{n}}$ is a linear transformation of the unit normal $\mathbf{n}$ by the Cauchy stress tensor $\boldsymbol{\sigma}$. Use of previous equations and the divergence theorem yields Cauchy first law of motion:

$$
\operatorname{div} \boldsymbol{\sigma}+\mathbf{b}=\rho \boldsymbol{\gamma}
$$

The second law of Eq. 0.15 together with Eq. 0.17 and Eq. 0.18 yields the equivalent local moment balance condition restricting the Cauchy stress $\boldsymbol{\sigma}$ to the space of symmetric tensors

$$
\boldsymbol{\sigma}=\boldsymbol{\sigma}^{T}
$$

The Cauchy stress characterizes the contact force distribution $\mathbf{t}_{\mathbf{n}}$ in $\kappa$ per unit current area in $\kappa$. But this is often inconvenient in solid mechanics because the deformed configuration generally is not known a priori. 
Therefore, the engineering stress tensor $\mathbf{T}_{R}$, also known as the first PiolaKirchhoff stress tensor, is introduced to define the contact force distribution $\mathbf{t}_{\mathbf{n}} \equiv \mathbf{T}_{R} \cdot \mathbf{N}$ in $\kappa$ per unit reference area in $\kappa_{R}$. Then for the same contact force $\mathrm{d} \mathscr{F}(\mathscr{P}, t)$, we must have:

$$
\mathrm{d} \mathscr{F}(\mathscr{P}, t) \equiv \mathbf{t}_{\mathbf{n}} \mathrm{d} a=\boldsymbol{\sigma} \cdot \mathbf{n} \mathrm{d} a=\mathbf{T}_{R} \cdot \mathbf{N} \mathrm{d} A=\mathbf{t}_{\mathbf{N}} \mathrm{d} A
$$

The vector $\mathbf{t}_{\mathbf{N}}$ is named the engineering stress tensor. We thus obtain the rule:

$$
\mathbf{T}_{R}=J \boldsymbol{\sigma} \mathbf{F}^{-T}
$$

relating the engineering and Cauchy stress tensors.

The corresponding stress principle and balance laws become:

$$
\mathbf{t}_{\mathbf{N}}=\mathbf{T}_{R} \cdot \mathbf{N}
$$

$$
\begin{gathered}
\operatorname{Div} \mathbf{T}_{R}+\mathbf{b}_{R}=\rho_{R} \gamma_{R} \\
\mathbf{T}_{R} \mathbf{F}^{T}=\mathbf{F} \mathbf{T}_{R}^{T}
\end{gathered}
$$

Hence the engineering stress $\mathbf{T}_{R}$ generally is not symmetric. Equation $\mathbf{b}_{R} \equiv J \mathbf{b}$ identifies the body force per unit volume in $\kappa_{R}$, and Div denotes the divergence operator with respect to $\mathbf{X}$ in $\kappa_{R}$, whereas div is with respect to $\mathbf{x}$ in $\kappa$.

Another stress tensor that will be usefull is the second Piola-Kirchhoff stress defined as:

$$
\boldsymbol{\pi}=\mathbf{F}^{-1} \mathbf{T}_{R}=J \mathbf{F}^{-1} \boldsymbol{\sigma} \mathbf{F}^{-T}
$$

Thus far, the deformation of a continuum and the actions that produce it have been treated separately without mention of any special material characteristics the body may possess. Of course the inherent constitutive nature of the material dictates its deformation response to action by forces and torques. For a specific class of materials, the specific relationship between the deformation gradient $\mathbf{F}$, the rate of deformation $\dot{\mathbf{F}}$ and the stress $\boldsymbol{\sigma}, \mathbf{T}_{R}$ or $\boldsymbol{\pi}$ is described by an equation known as a constitutive equation. In the next section, the principle of balance of mechanical energy will be applied to derive the constitutive equation for a special class of perfectly elastic materials called hyperelastic solids. 


\subsection{Hyperelasticity}

Thermodynamics foundation The first law of thermodynamics tells that the time rate of change of the internal energy $E(\mathscr{P}, t)$ for any part $\mathscr{P} \subset \mathscr{B}$ of a body $\mathscr{B}$ is balanced by the total mechanical power $W(\mathscr{P}, t)$ and the total heat flux $Q(\mathscr{P}, t)$.

$$
\dot{E}(\mathscr{P}, t)=W(\mathscr{P}, t)+Q(\mathscr{P}, t)
$$

The second law of thermodynamics tells that the time rate of change of entropy $\dot{S}(\mathscr{P}, t)$ for any part $\mathscr{P} \subset \mathscr{B}$ of a body $\mathscr{B}$ can be decomposed into exchanges of entropy and production of entropy and that the latter can only be positive, or zero if the transformation is reversible (no dissipation). If $\Theta$ denotes temperature, exchanges of entropy at constant temperature (isotherm transformations will be assumed further) may be written such as: $Q / \Theta$. Finally, the second law of thermodynamics tells:

$$
\begin{gathered}
\dot{S}(\mathscr{P}, t) \geq \frac{Q(\mathscr{P}, t)}{\Theta} \\
\dot{E}(\mathscr{P}, t)-\Theta \dot{S}(\mathscr{P}, t) \leq W(\mathscr{P}, t)
\end{gathered}
$$

$\mathscr{P}$ being an arbitrary tetrahedral element, and $\boldsymbol{\sigma}: \mathbf{D}$ being the mechanical power per unit volume, it may be written at any time $t$ :

$$
\rho(\dot{e}-\Theta \dot{s}) \leq \boldsymbol{\sigma}: \mathbf{D}
$$

where $e$ denotes the local specific internal energy and $s$ denotes the local specific entropy. This equation points out that the work done by the stress would induce either an increase of the specific internal energy or a decrease of the specific entropy. In the case of elasticity, the transformation is reversible and it may be written:

$$
\rho(\dot{e}-\Theta \dot{s})=\boldsymbol{\sigma}: \mathbf{D}
$$

When the work done by the stress induces mostly an increase of the specific internal energy $(|\Theta \dot{s}|<<\dot{e}$ ), we speak of enthalpic elasticity (in an isotherm transformation, $\dot{e}=\dot{\mathrm{h}}$ where $\mathrm{h}$ would be the specific enthalpy). Enthalpic elasticity is the elasticity of cristals where the deformation comes mostly from a change of distances between atoms. Elastic response of the crystalline solids is due to the change of the equilibrium interatomic distances under stress and therefore, the change in the internal energy of the crystal. 
When the work done by the stress induces mostly a decrease of entropy $(\dot{e}<<|\Theta \dot{s}|)$, we speak of entropic elasticity. Elasticity of soft biological tissues is composed from the elastic responses of the chains crosslinked in the network sample. External stress changes the equilibrium end-to-end distance of a chain, and it thus adopts a less probable conformation, its entropy therefore decreases. Therefore, the elasticity of soft biological tissues is of purely entropic nature.

Introducing the specific free energy $\phi=e+\Theta s$, and still assuming isotherm transformations $(\dot{\Theta}=0)$, it may be written:

$$
\rho \dot{\phi}=\sigma: \mathbf{D}
$$

It is now the time to define what a hyperelastic solid is. A hyperleastic solid is a material whose specific free energy depends only on the strain. It may be written:

$$
\phi(\mathbf{X}, t)=\phi(\mathbf{F}(\mathbf{X}, t), \mathbf{X})
$$

The $\psi=\rho \phi$ function is a strain energy density function. Then the constitutive equation for a hyperelastic solid can be written:

$$
\begin{gathered}
\boldsymbol{\pi}=\frac{\partial \psi}{\partial \mathbf{E}}=2 \frac{\partial \psi}{\partial \mathbf{C}} \\
\mathbf{T}_{R}=\frac{\partial \psi}{\partial \mathbf{F}}=\mathbf{F} \frac{\partial \psi}{\partial \mathbf{E}}=2 \mathbf{F} \frac{\partial \psi}{\partial \mathbf{C}} \\
\boldsymbol{\sigma}=J^{-1} \frac{\partial \psi}{\partial \mathbf{F}} \mathbf{F}^{T}=J^{-1} \mathbf{F} \frac{\partial \psi}{\partial \mathbf{E}} \mathbf{F}^{T}=2 J^{-1} \mathbf{F} \frac{\partial \psi}{\partial \mathbf{C}} \mathbf{F}^{T}
\end{gathered}
$$

Isotropic compressible hyperelastic solids For an isotropic solid, the strain energy function must be an isotropic scalar valued function of the principal invariants alone:

$$
\psi=\psi(\mathbf{C})=\psi(\mathbf{B})=\psi\left(I_{1}, I_{2}, I_{3}\right)
$$

wherein, specifically,

$$
\begin{gathered}
I_{1}=\operatorname{tr}(\mathbf{B}) \\
I_{2}=\frac{1}{2}\left[I_{1}^{2}-\operatorname{tr}\left(\mathbf{B}^{2}\right)\right] \\
I_{3}=\operatorname{det}(\mathbf{B})
\end{gathered}
$$


Note that Eq. 0.35 may be rewritten such as:

$$
\boldsymbol{\sigma}=2 J^{-1} \frac{\partial \psi}{\partial \mathbf{B}} \mathbf{B}
$$

Then, introducing the principal invariants:

$$
\boldsymbol{\sigma}=\left(\frac{\partial \psi}{\partial J}+2 J^{-1} \frac{\partial \psi}{\partial I_{2}} I_{2}\right) \mathbf{1}+2 J^{-1} \frac{\partial \psi}{\partial I_{1}} \mathbf{B}-2 J^{-1} \frac{\partial \psi}{\partial I_{-1}} \mathbf{B}^{-1}
$$

where $I_{-1}=I_{2} / I_{3}=\operatorname{tr}\left(\mathbf{B}^{-1}\right)$.

Isotropic hyperelastic incompressible solids The Cauchy stress on an incompressible, hyperelastic material, is determined by $\mathbf{F}$ only to within an arbitrary stress which is proportional to the identity tensor. Then the constitutive equation for an incompressible, isotropic, hyperelastic material is given by:

$$
\boldsymbol{\sigma}=-p \mathbf{1}+2 \frac{\partial \psi}{\partial I_{1}} \mathbf{B}-2 \frac{\partial \psi}{\partial I_{2}} \mathbf{B}^{-1}
$$

where $p$ is an undetermined scalar of $\mathbf{x}$. Note that $I_{2}=I_{-1}$ for an incompressible solid.

A particular type of strain energy functions may be written such as polynomials:

$$
\psi=\sum_{j=0}^{N_{j}} \sum_{i=0}^{N_{i}} C_{i j}\left(I_{1}-3\right)^{i}\left(I_{2}-3\right)^{j}
$$

when $N_{i}=3$ and $N_{j}=0$ it is referred to as Yeoh strain energy function, when $N_{i}=1$ and $N_{j}=1$ but $C_{11}=0$, we have the Mooney-Rivlin material. The special case when $N_{i}=1$ and $N_{j}=0$ is the Neo-Hookean material.

Another particular type which is meaningful for biological tissues may be written:

$$
\psi=\frac{\mu_{0}}{2 \gamma}\left[e^{\gamma\left(I_{1}-3\right)}-1\right]
$$

Isotropic hyperelastic nearly incompressible solids It is common for nearly incompressible hyperelastic solids to assume a perfect decoupling between purely volumetric and purely isochoric effects, and then to decompose the strain energy density function additively in two components: one depending only on volume changes and the second one independent of volume changes: 


$$
\psi=U(J)+\bar{\psi}\left(\bar{I}_{1}, \bar{I}_{2}\right)
$$

$$
\begin{aligned}
\boldsymbol{\sigma}=\left(\frac{\partial \psi}{\partial J}+2 J^{-1} \frac{\partial \psi}{\partial I_{2}}\right. & \left.I_{2}\right) \mathbf{1} \\
& +2 J^{-1} \frac{1}{J^{2 / 3}} \frac{\partial \bar{\psi}}{\partial \bar{I}_{1}} \mathbf{B}-2 J^{-1} \frac{1}{J^{4 / 3} / J^{2}} \frac{\partial \bar{\psi}}{\partial \bar{I}_{2}} \mathbf{B}^{-1}
\end{aligned}
$$

where $\bar{I}_{1}=\operatorname{tr}(\overline{\mathbf{B}}), \bar{I}_{2}=\frac{1}{2}\left[\bar{I}_{1}^{2}-\operatorname{tr}\left(\overline{\mathbf{B}}^{2}\right)\right]=\operatorname{tr}\left(\overline{\mathbf{B}}^{-1}\right), \overline{\mathbf{B}}=\overline{\mathbf{F}} \overline{\mathbf{F}}^{T}$ and $\overline{\mathbf{F}}=$ $J^{-1 / 3} \mathbf{F}$.

$$
\begin{gathered}
\boldsymbol{\sigma}=\left[\left(\frac{\partial \psi}{\partial J}+2 J^{-1} \frac{\partial \psi}{\partial I_{2}} I_{2}\right)+\frac{\partial \bar{\psi}}{\partial \bar{I}_{1}} \frac{\partial \bar{I}_{1}}{\partial J}+\frac{\partial \bar{\psi}}{\partial \bar{I}_{2}} \frac{\partial \bar{I}_{2}}{\partial J}\right] \mathbf{1} \\
+2 J^{-1} \frac{\partial \bar{\psi}}{\partial \bar{I}_{1}} \overline{\mathbf{B}}-2 J^{-1} \frac{\partial \bar{\psi}}{\partial \bar{I}_{2}} \overline{\mathbf{B}}^{-1}
\end{gathered}
$$

Finally,

$$
\begin{aligned}
\boldsymbol{\sigma}=\left[\frac{\partial \psi}{\partial J}-\frac{2}{3} J^{-1} \frac{\partial \bar{\psi}}{\partial \bar{I}_{1}} \bar{I}_{1}\right. & \left.+\frac{2}{3} J^{-1} \frac{\partial \bar{\psi}}{\partial \bar{I}_{2}} \bar{I}_{2}\right] \mathbf{1}+2 J^{-1} \frac{\partial \bar{\psi}}{\partial \bar{I}_{1}} \overline{\mathbf{B}}-2 J^{-1} \frac{\partial \bar{\psi}}{\partial \bar{I}_{2}} \overline{\mathbf{B}}^{-1} \\
& =\frac{\partial \psi}{\partial J} \mathbf{1}+2 J^{-1} \operatorname{Dev}\left(\frac{\partial \bar{\psi}}{\partial \bar{I}_{1}} \overline{\mathbf{B}}-\frac{\partial \bar{\psi}}{\partial \bar{I}_{2}} \overline{\mathbf{B}}^{-1}\right)
\end{aligned}
$$

where Dev denotes the deviatoric tensor. 
The Cauchy stress is then decomposed additively into a hydrostatic component related to $J$ and into a deviatoric component related to $\bar{I}_{1}$ and $\bar{I}_{2}$.

$$
\boldsymbol{\sigma}=-p \mathbf{1}+\mathbf{s}
$$

where $p=-\partial U / \partial J$ and

$$
\mathbf{s}=2 J^{-1} \operatorname{Dev}\left(\frac{\partial \bar{\psi}}{\partial \bar{I}_{1}} \overline{\mathbf{B}}-\frac{\partial \bar{\psi}}{\partial \bar{I}_{2}} \overline{\mathbf{B}}^{-1}\right)
$$

Common models of isotropic hyperelastic nearly incompressible solids The compressible version of a Neo-Hookean material may be written:

$$
\psi=C_{10}\left(\bar{I}_{1}-3\right)+\frac{1}{D}(J-1)^{2}
$$

The compressible version of a Yeoh material may be written:

$$
\psi=\sum_{i=0}^{3} C_{i 0}\left(\bar{I}_{1}-3\right)^{i}+\sum_{i=0}^{3} \frac{1}{D_{i}}(J-1)^{2 i}
$$

Another common model in compressible hyperelasticity is the Arruda Boyce model. Although its formulation is based on a thermondynamical background, it is not often used for biological tissues. The strain energy density may be written:

$$
\psi=\mu \sum_{i=0}^{5} \frac{C_{i}}{\lambda_{m}^{2 i-2}}\left(\bar{I}_{1}^{i}-3^{i}\right)+\frac{1}{D}\left[\frac{\left(J^{2}-1\right)}{2}-\ln (J)\right]
$$

where: $C_{1}=\frac{1}{2}, C_{2}=\frac{1}{20}, C_{3}=\frac{11}{1050}, C_{4}=\frac{19}{7050}, C_{5}=\frac{51}{673750}$.

A more common model is the Ogden model, which may be written:

$$
\psi=\frac{2 \mu}{\alpha^{2}}\left(\lambda_{1}^{2 \alpha}+\lambda_{2}^{2 \alpha}+\lambda_{3}^{2 \alpha}\right)+\frac{1}{D}\left(J^{2}-1\right)
$$

where $\lambda_{1}, \lambda_{2}$ and $\lambda_{3}$ are the principal stretches.

\subsection{More sophisticated constitutive models}

The aim of this section is to introduce the basics for the following sections of this chapter but also for the following chapters of this book. It is not rare that soft tissues are modeled with constitutive equations including other features than the ones of isotropic hyperelasticity. The main ones are summarized hereafter. 
Anisotropic hyperelastic models Soft tissues may often present anisotropic effects. The most common effect is a different stress-stretch curve when they are subjected to uniaxial tension in two different directions. Very common models permitting to represent these effects may describe the material such as a composite made of a Neo-Hookean matrix in which fiber families are embedded:

$$
\psi=C_{10}\left(\bar{I}_{1}-3\right)+\sum_{i=1}^{N} \frac{k_{1 i}}{2 k_{2 i}}\left[e^{k_{2 i}\left(\bar{\lambda}_{i}^{2}-1\right)}-1\right]+\frac{1}{D}\left(J^{2}-1\right)
$$

where $\bar{\lambda}_{i}^{2}=\overline{\mathbf{C}}:\left(\mathbf{M}_{\mathbf{i}} \otimes \mathbf{M}_{\mathbf{i}}\right)=\overline{\mathbf{C}} \mathbf{M}_{\mathbf{i}} \cdot \mathbf{M}_{\mathbf{i}}$.

$\mathbf{M}_{\mathbf{i}}$ are vectors defining orientations of a fiber family in the reference configuration. Although motivated by microstructural information, this type of models were developed primarily to capture phenomenologically the anisotropic response of soft tissues subjected to multi-directional tensile tests, which ultimately depends on constituent fractions, fiber orientations, cross-linking, physical entanglements, and so forth.

Irreversible effects When subjected to cycled uniaxial tensile tests (or other types of testing), the loading unloading profile of biological tissues often presents an hysteresis on the first cycle. With repeated loading cycles the load-deformation curves shift to the right in a load-elongation diagram and the hysteretic effects diminish. In a load-time diagram the load-time curves shift upwards with increasing repetition number. By repeated cycling, eventually a steady state is reached at which no further change will occur unless the cycling routine is changed. In this state the tissue is said to be preconditioned. Any change of the lower or upper limits of the cycling process requires new preconditioning of the tissue. Preconditioning occurs due to internal changes in the structure of the tissue. Hysteresis, non-linearity, relaxation and preconditioning are common properties of all soft tissues, although their observed degrees vary.

The difference between the loading and unloading response can be simulated using an isotropic damage formulation. It consists in writing the strain energy in the form of:

$$
\psi=(1-d) \bar{\psi}+\frac{1}{D}\left(J^{2}-1\right)
$$

where $1-d$ is a reduction factor and $d$ is a scalar damage variable defined in $0 \leq d \leq 1$. When $d=0$ the material is undamaged. The value $d=1$ is an upper limit in which the material is completely damaged and failure occurs. 
The evolution of damage may be described by a function of a maximum equivalent strain defined such as $\zeta^{m}=\max _{t \in]-\infty, t]} \sqrt{2 \overline{\psi(\mathbf{E}(t)}}$ where $\mathbf{E}(t)$ is the Green-Lagrange strain tensor for the pseudo time $t$ of the deformation process. The evolution of damage can be described with an exponential form:

$$
1-d\left(\zeta^{m}\right)=\beta+(1-\beta) \frac{1-e^{-\zeta^{m} / \alpha}}{\zeta^{m} / \alpha}
$$

where $\alpha$ and $\beta$ are material parameters.

Damage can also be modeled with the concept of softening hyperelasticity. In this concept, instead of having a strain energy tending to infinity when the norm of the stretch tensor tends to infinity, the stored energy is bounded [2].

More details about damage models are given in Chapter 4 of this book.

Time dependent effects The hysteresis in the stress-strain relationship may also show the viscoelastic behaviour of soft biological tissue. The simplest model of viscoelasticity is the Kelvin model combining a linear spring and a dashpot. In analogy to linear viscoelasticity in small strain, we can assume an additive free energy potential with the form $\psi=\psi_{0}+\psi_{v}$ where $\psi_{0}$ measures the energy stored in the elastic branch (equilibrium) and $\psi_{v}$ measured the energy stored in the viscous branch, which progressively disappears during relaxation.

In a viscoelastic material the history of strain affects the actually observed stress. As well, loading and unloading occur on different stress-strain paths. The hysteresis of most biological tisssues is assumed to show only little dependence on the strain rate within several decades of strain rate variation. This insensitivity to strain rate over several decades is not compatible with simple viscoelastic models consisting for instance of a single spring and dashpot element. With such a simple viscoelasticity approach the material model will show a maximum hysteresis loop at a certain strain rate whereas all other strain rates will show a smaller hysteresis loop. A model consisting of a discrete number of spring-dashpot elements therefore produces a discrete hysteresis spectrum with maximum dissipation at discrete strain rates. It may be written such:

$$
\bar{\psi}=\int_{0}^{t}\left[\left(1-\sum_{k=1}^{N} g_{k}\left(1-e^{-\frac{t-\tau}{\tau_{k}}}\right)\right) \times \frac{d \psi_{0}}{d \tau}\right] d \tau
$$

where $\tau_{k}$ are the relaxation times and $g_{k}$ are the relaxation coefficients. 
It is widely accepted that soft connective tissues are multiphasic materials. They are sometimes modeled as a mixture of two immiscible constituents: an solid hyperelastic matrix and an interstitial incompressible fluid. This type of models, sometimes called poroelastic models, can particularly describe both the stress distribution and interstitial fluid motion within the cartilage tissue under various loading conditions. Moreover the interaction between the solid and the fluid phases has been identified to be responsible for the apparent viscoelastic properties in the compression of hydrated soft tissues.

Active models It is often assumed that in the presence of an actin-myosin complex in the soft tissue, the total Cauchy stress can be split into two parts: $\boldsymbol{\sigma}=\boldsymbol{\sigma}_{p}+\boldsymbol{\sigma}_{a}$, where $\boldsymbol{\sigma}_{p}$ and $\boldsymbol{\sigma}_{a}$ denote passive and active stress respectively. The passive stress results from the elastic deformation of the tissue and can be derived from the theory of hyperelasticity. The active stress is generated in myofibrils or in smooth muscle cells by activation and is directed parallel to the fibre orientation. Hence: $\boldsymbol{\sigma}_{a}=\sigma_{a} \boldsymbol{\epsilon} \otimes \boldsymbol{\epsilon}$ where $\boldsymbol{\epsilon}$ is the unit vector identifying the orientation. The mechanism for generating $\sigma_{a}$ involves internal variables.

\subsection{Growth and remodelling models}

Many experiments have shown that the stress field dictates, at least in part, the way in which the microstructure of soft tissues is organized. This observation leads to the concept of functional adaptation wherein it is thought that soft tissues functionally adapt so as to maintain particular mechanical metrics (e.g., stress) near target values. To accomplish this, tissues often develop regionally varying stiffness, strength and anisotropy.

Models of growth and remodelling necessarily involve equations of reactiondiffusion. There has been a trend to embed the reactiondiffusion framework within tissue mechanics $[3,4]$. The primary assumption is that one models volumetric growth through a growth tensor $\mathbf{F}_{g}$, which describes changes between two fictitious stress-free configurations: the original body is imagined to be fictitiously cut into small stress-free pieces, each of which is allowed to grow separately via $\mathbf{F}_{g}$, with $\operatorname{det}\left(\mathbf{F}_{g}\right) \neq 1$. Because these growths need not be compatible, internal forces are often needed to assemble the grown pieces, via $\mathbf{F}_{a}$, into a continuous configuration. This, in general, produces residual stresses, which are now known to exist in many soft tissues. The formulation is completed by considering elastic deformations, via $\mathbf{F}_{a}$, from the intact but residually stressed traction-free configuration to a current configuration that is induced by external mechanical loads. 
The initialboundary value problem is solved by introducing a constitutive relation for the stress response to the deformation $\mathbf{F}_{e} \mathbf{F}_{a}$, which is often assumed to be incompressible hyperelastic, plus a relation for the evolution of the stress-free configuration via $\mathbf{F}_{g}$. Thus, growth is assumed to occur in stress-free configurations and typically not to affect material properties.

Although the previous theory called the theory of kinematic growth yields many reasonable predictions, Humphrey and coworkers have suggested that it models consequences of growth and remodelling, not the processes by which they occur. Growth and remodelling necessarily occur in stressed, not fictitious stress-free, configurations, and they occur via the production, removal, and organization of different constituents; moreover, growth and remodelling need not restore stresses exactly to homeostatic values. Hence, Humphrey and coworkers introduced a conceptually different approach to model growth and remodelling, one that is based on tracking the turnover of individual constituents in stressed configurations (the constrained mixture model $[5,6])$

\section{Characterization of hyperelastic properties using a bulge inflation test}

After the introduction of basics about nonlinear finite-strain constitutive relations, we now introduce approaches of experimental biomechanics and inverse methods aimed at quantifying constitutive parameters of soft tissues.

\subsection{Introduction}

Traditional characterization of material constants in hyperelastic solids The hyperelastic constants in the strain energy density function of a material determine its mechanical response. For identifying these hyperelastic materials, simple deformation tests (consisting of six deformation models - see Figure 0.1) can be used. It is always recommended to take the data from several modes of deformation over a wide range of strain values.

Even though the superposition of tensile or compressive hydrostatic stresses on a loaded incompressible body results in different stresses, it does not alter deformation of a material. Upon the addition of hydrostatic stresses, the following modes of deformation are found to be identical: uniaxial tension and equibiaxial compression, uniaxial compression and equiaxial tension, and planar tension and planar compression. It reduces to 3 independent deformation states for which we can obtain experimental data.

For each of the three independent tests, the resultant force $F$ can be 
expressed analytically with respect to the applied stretch $\lambda$ using the following formulas of incompresssible hyperelasticity which are derived from the equations introduced above:

1. in uniaxial tension:

$$
F=2 S_{0}\left(\lambda-\lambda^{-3}\right)\left(\frac{\partial \psi}{\partial I_{1}}+\frac{\partial \psi}{\partial I_{2}}\right)
$$

2. in planar tension:

$$
F=2 S_{0}\left(\lambda-\lambda^{-3}\right)\left(\lambda \frac{\partial \psi}{\partial I_{1}}+\frac{\partial \psi}{\partial I_{2}}\right)
$$

3. in equibiaxial tension:

$$
F=2 S_{0}\left(\lambda-\lambda^{-5}\right)\left(\frac{\partial \psi}{\partial I_{1}}+\lambda^{2} \frac{\partial \psi}{\partial I_{2}}\right)
$$

where $S_{0}$ is the initial cross sectional area of the sample.

The identification of the material constants is achieved by a least-squares fit analysis which consists in minimizing the sum of squared discrepancies between the experimental values (if any) of $F$ and the values predicted by the models. This yields a set of simultaneous equations which are solved for the material constants.

The identification of material constants is seldom achieved on cylindrical specimens where analytical formulas can also be derived to perform again least-squares fit analysis [7].

The bulge inflation test combined with digital image correlation As introduced previously, traditional characterization of material constants in hyperelastic solids is based on a least-squares fit analysis of $F$ versus $\lambda$ curves. In the tests, $\lambda$ is usually measured using traditional extensometry techniques being either based on tracking the motion of the grips in the machine used to apply the deformation on the tissue, sometimes based on tracking the motion of markers or dots drawn on the tissue itself.

Recently, it has become a common practice to combine video based fullfield displacement measurements experienced by tissue samples in vitro, with custom inverse methods to infer (using nonlinear regression) the best-fit material parameters and the rupture stresses and strains. These approaches offer important possibilities for fundamental mechanobiology research as they permit to quantify regional variations in properties in situ.

Here we present an illustrative example of the author's experience where bulge inflation tests are carried out on aneurysm samples for characterzing the regional variations of hyperelastic constants across them. 


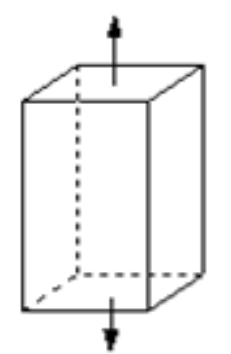

Uniaxial Tension

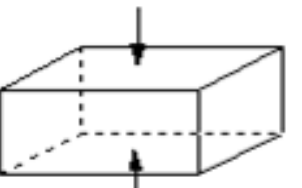

Uniaxial Compression

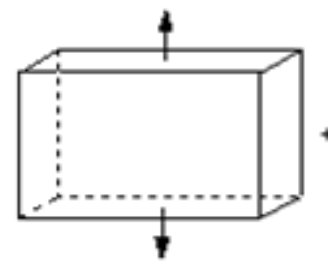

Planar Tension

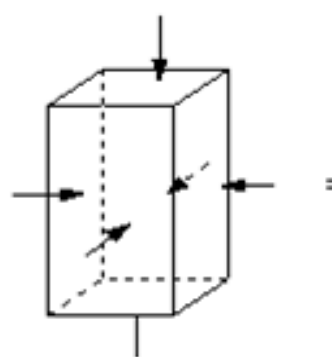

Hydrostatic Compression

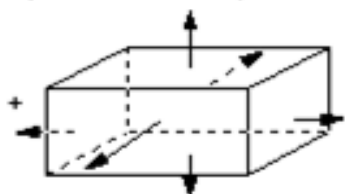

Hydrostatic Tension

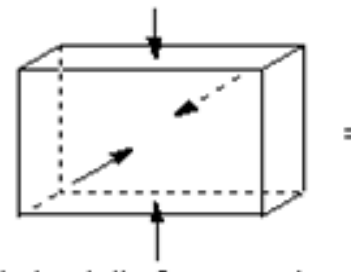

Hydrostatic Compression (Plane Strain Assumption)

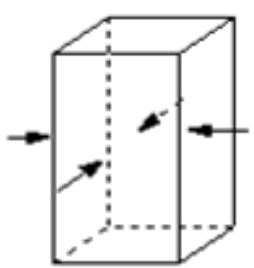

Equibiaxial Compression

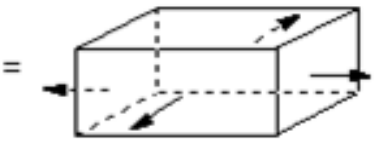

Equibiaxial Tension

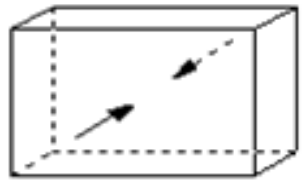

Planar Compression

Figure 0.1: Schematic representation of independent testing modes for hyperelastic materials.

\subsection{Materials and methods}

Experimental arrangements The study deals with the characterization of aortic tissues collected on patients having an ascending thoracic aortic aneurysm (ATAA). In this reported example, an unruptured ATAA section was collected from a patient undergoing elective surgery to replace his ATAA with a graft in accordance with a protocol approved by the Institutional Review Board of the University Hospital Center of Saint-Etienne. After retrieval, the specimen was placed in saline solution and stored at $4^{\circ} \mathrm{C}$ until testing, which occurred within 48 hours of the surgery. Immediately prior to testing, the ATAA was cut into a square specimen approximately $45 \times$ $45 \mathrm{~mm}$. Any fatty deposits were removed from the surface of the tissue to ensure that during mechanical testing the tissue did not slip in the clamps. 


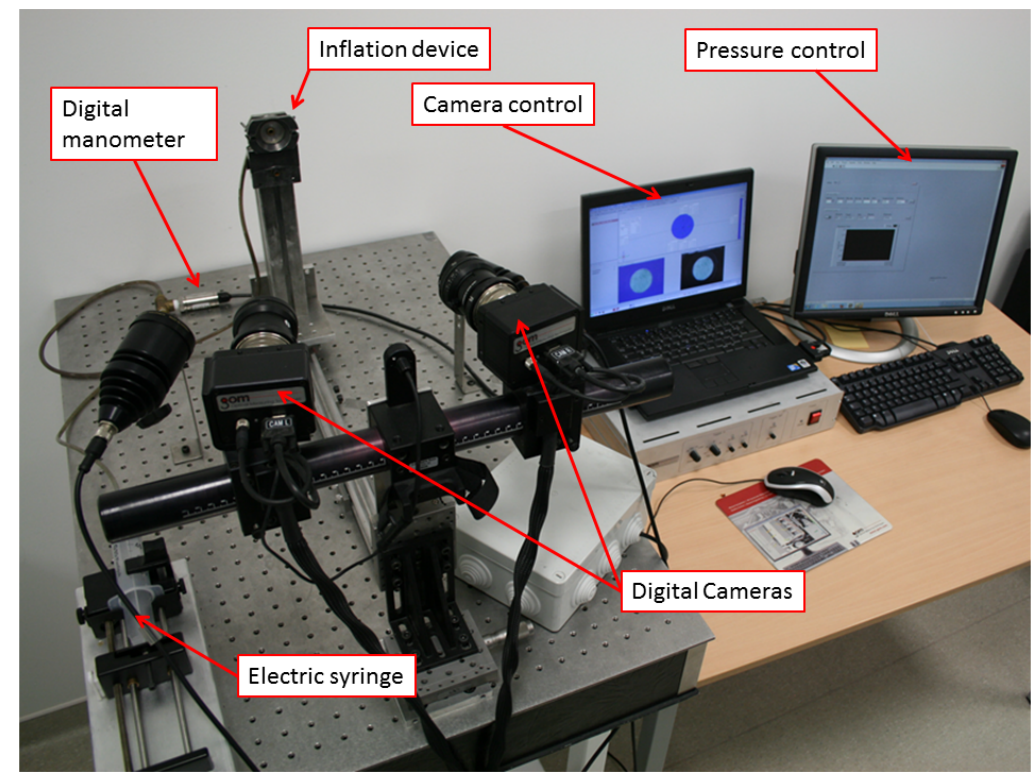

(a)
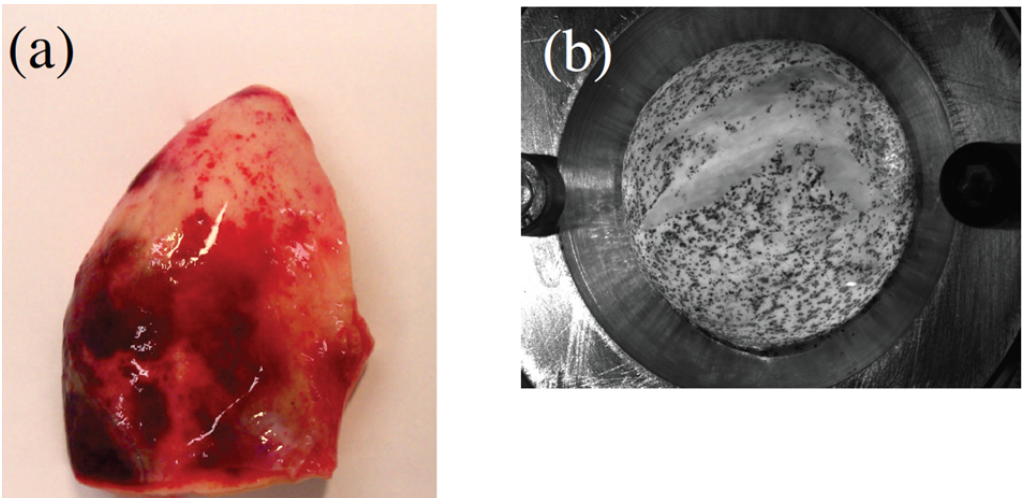

Figure 0.2: Experimental setup and test sample (a) before testing and (b) after rupture.

An average thickness was found for the sample by measuring the thickness of the tissue at a minimum of 5 locations.

The specimen was clamped in the bulge inflation device, Figure 0.2, so that the luminal side of the tissue faced outward. Then a speckle pattern was applied to the luminal surface using black spray paint. The sample was 
inflated using a piston driven at $15 \mathrm{~mm} / \mathrm{min}$ to infuse water into the cavity behind the sample. During the test, the pressure was measured using a digital manometer (WIKA, DG-10). Images of the inflating specimen were collected using a commercial DIC system (GOM, 5M LT) composed of two 8-bit CCD cameras equipped with $50 \mathrm{~mm}$ lenses (resolution: $1624 \times 1236$ px). The cameras were positioned $50 \mathrm{~cm}$ apart at an angle of $30^{\circ}$ with an aperture of $f / 11$. This produced a depth of field of $15.4 \mathrm{~mm}$ which was sufficient to capture the deformation of the tissue up to failure. Images of the deforming sample were collected every $3 \mathrm{kPa}$ until the sample ruptured.

After rupture, the collected images were analyzed using the commercial correlation software ARAMIS (GOM, v. 6.2.0) to determine the three dimensional displacement of the tissue surface. For the image analysis, a facet size of 21 px and a facet step of 5 px were chosen based on the speckle pattern dot size, distribution, and contrast. The selected parameters produced a cloud of approximately 15,000 points where the three displacement values were calculated. Details about the error quantification of the method may be found in the original paper [8].

Geometric Reconstruction A deforming NURBS mesh was extracted by morphing a NURBS template to the DIC point clouds. The template was a circular domain with a diameter slightly less than that of the point cloud in the first pressure state. The NURBS surface was parameterized as a single patch containing clamped knots of 20 divisions in each parametric direction, with $22 \times 22$ control points. Since NURBS control points, in general, do not fall on the surface they describe, they cannot be directly derived from the DIC clouds. Instead, the positions of the Gauss points were obtained first using the moving least square method [9]. For each Gauss point, a set of nearest image points in the DIC point cloud were identified based on their distance to the Gauss point in the first pressure state. The radius of the neighboring region was automatically adjusted to that it contained at least 6 image points. The position of each Gauss point, $\mathbf{y}_{g}$, was computed using an affine interpolation

$$
\mathbf{y}_{g}=\frac{\sum_{\mathbf{y} \in \Omega_{g}} w_{j} \mathbf{y}_{j}}{\sum_{\mathbf{y} \in \Omega_{g}} w_{j}}
$$

where $\mathbf{y}_{j}$ is the position vector for each image point in the neighborhood, $\Omega_{g}$, and $w_{i}$ is the weighting function taken to be the inverse of the distance from $\mathbf{y}_{j}$ to the Gauss point. Using the same weights calculated in the first stage, the Gauss points in every pressure stage were identified.

A global least squares problem was then formulated to compute the best- 
fit positions of the control points. The NURBS surface was represented as

$$
\mathbf{x}=\sum_{i} N_{i}\left(u_{1}, u_{2}\right) \mathbf{Q}_{i}
$$

where $N_{i}$ are the NURBS basis functions, $\mathbf{Q}_{i}$ are the control points, and the pair of knot variables, $\left(u_{1}, u_{2}\right)$, represent a material point. The position of a modeled Gauss point is then given by $\mathbf{x}_{g}=\sum_{i} N_{i}\left(u_{1 g}, u_{2 g}\right) \mathbf{Q}_{i}$. The position of the control points were obtained by minimizing a weighted sum of $\left\|\mathbf{x}_{g}-\mathbf{y}_{g}\right\|^{2}$ over all Gauss points. This procedure was applied to each pressure state.

The accuracy of this reconstruction method was previously assessed and showed by [10].

Strain reconstruction Surface strains were computed in the local NURBS curvilinear coordinate system. The surface coordinates, $u_{\alpha},(\alpha, \beta=1,2)$ induce a set of convected basis vectors $\left(\mathbf{a}_{1}, \mathbf{a}_{2}\right)$ where $\mathbf{a}_{\alpha}=\frac{\partial \mathbf{x}}{\partial \mathbf{u}_{\alpha}}$ and $\mathbf{x}\left(u_{1}, u_{2}\right)$ is the NURBS representation given in Eq. 0.65. The reciprocal basis $\left(\mathbf{a}^{1}, \mathbf{a}^{2}\right)$ are computed such that $\mathbf{a}_{\alpha} \cdot \mathbf{a}_{\beta}=\delta_{\alpha \beta}$. In the reference configuration, the basis vectors are denoted by $\left(\mathbf{A}_{1}, \mathbf{A}_{2}\right)$ and $\left(\mathbf{A}^{1}, \mathbf{A}^{2}\right)$.

The surface deformation gradient tensor is

$$
\mathbf{F}=\mathbf{a}_{\alpha} \otimes \mathbf{A}^{\alpha} .
$$

It then follows that the surface Cauchy-Green deformation tensor, $\mathbf{C}$, and the Green-Lagrangian strain tensor, $\mathbf{E}$, are given by

$$
\begin{gathered}
\mathbf{C}=\left(\mathbf{a}_{\alpha} \cdot \mathbf{a}_{\beta}\right) \mathbf{A}^{\alpha} \otimes \mathbf{A}^{\beta} \\
\mathbf{E}=\frac{1}{2}\left(\mathbf{a}_{\alpha} \cdot \mathbf{a}_{\beta}-\delta_{\alpha \beta}\right) \mathbf{A}^{\alpha} \otimes \mathbf{A}^{\beta} .
\end{gathered}
$$

The physical components of $\mathbf{C}$ and $\mathbf{E}$ are computed by identifying a local orthonormal basis $\left(\mathbf{G}_{1}, \mathbf{G}_{2}\right)$ that is constructed in the tangent plane spanned by $\left(\mathbf{A}_{1}, \mathbf{A}_{2}\right)$. The physical components of the Cauchy-Green deformation tensor, $C_{\alpha \beta}$, and Green-Lagrangian strain tensor, $E_{\alpha \beta}$, are $C_{\alpha \beta}=\mathbf{G} \cdot \mathbf{C} \mathbf{G}$ and $E_{\alpha \beta}=\mathbf{G} \cdot \mathbf{E} \mathbf{G}$, respectively.

Wall stress reconstruction For an inverse membrane boundary value problem the deformed configurations and boundary conditions are given as inputs to the FE model and the wall stress is calculated. The balance equation that governs static equilibrium is $[11,12]$

$$
\frac{1}{\sqrt{\mathrm{a}}}\left(\sqrt{\mathrm{a}} t^{\alpha \beta} \mathbf{a}_{\alpha}\right)_{, \beta}+p \mathbf{n}=0
$$


where $\mathbf{a}$ is $\operatorname{det}\left(\mathbf{a}_{\alpha} \mathbf{a}_{\beta}\right), \mathbf{t}$ is the Cauchy wall tension, $p$ is the applied internal pressure, $\mathbf{n}$ is an outward facing unit normal, and ()$_{, \beta}$ indicates $\frac{\partial}{\partial u^{\beta}}$. Note that the Cauchy wall tension $\mathbf{t}$ is directly related to the Cauchy stress, $\boldsymbol{\sigma}$, through the current thickness of the membrane, $h$, via $t^{\alpha \beta}=h \sigma^{\alpha \beta}=t^{\beta \alpha}$.

The weak form of the boundary value problem reads

$$
\int_{\Omega} t^{\alpha \beta} \mathbf{a}_{\alpha} \cdot \delta \mathbf{x}_{, \beta} \mathrm{d} a-\int_{\Omega} p \mathbf{n} . \delta \mathbf{x} \mathrm{d} a=0
$$

where $\delta \mathbf{x}$ is any admissible variation to the current configuration $\Omega$. The details of the FE procedure for solving Eq. 0.70 were presented in [13]. Briefly, the Cauchy wall tension is regarded as a function of the inverse deformation gradient. The weak form subsequently yields a set of nonlinear algebraic equations for the positions of control points in the reference configuration. At the same time, the tension field in the current state is determined. An auxiliary material model is needed to perform the inverse analysis. The material model influences the predicted undeformed configuration; however, due to the static determinacy of Eq. 0.69, the influence is weak $[13,14,15,16]$. As in a previous study [17], a neo-Hookean model was implemented. For computational efficiency, the stiffness parameter of the model was set to unrealistically high values to ensure a robust convergence.

To simulate the experimental boundary conditions the outermost edge of the specimen was fixed. This boundary condition was applied directly to the control points on the outer boundary of the mesh. Applying any displacement based constraint in the inverse membrane analysis creates a boundary region in the solution where the stresses are inaccurate [13]. To minimize the influence of boundary effect, the outer ring of elements was excluded from further analyses. Since the influence region of each control point spans three elements in each of the two parametric directions, the outer three rings of elements were deemed to be the boundary region. By a retrospective comparison with the forward analysis reported the size of the boundary region was confirmed [18].

Material Property Identification Using inverse membrane analysis, the stress was calculated at every Gauss point. Combining the stress data with the local surface strains calculated from Eqs. 0.66 - 0.68, the stressstrain response at every Gauss point in the mesh is known. The local material properties at each Gauss point were then identified by fitting the local stress-strain response to a hyperelastic surface energy density. A anisotropic strain energy function was used, this anisotropy being implemented on the principle of Eq. 0.57. More specifically here, we used a modified form of the strain energy density proposed by Gasser, Ogden, and Holzapfel (GOH) 
[19] which may be written such as:

$$
w=\frac{\mu_{1}}{2}\left(\mathfrak{I}_{1}-\ln \left(\mathfrak{I}_{2}\right)-2\right)+\frac{\mu_{2}}{4 \gamma}\left(\mathrm{e}^{\gamma\left(\mathfrak{I}_{k}-1\right)^{2}}-1\right)
$$

where $\mathfrak{I}_{1}=\operatorname{tr} \mathbf{C}$ and $\mathfrak{I}_{2}=\operatorname{det} \mathbf{C}$ are the principal invariants of the CauchyGreen deformation tensor and $\mathfrak{I}_{\kappa}=\mathbf{C}:(\kappa \mathbf{1}+(1-2 \kappa) \mathbf{M} \otimes \mathbf{M})$ is a compound invariant consisting of isotropic and anisotropic contributions.

Litteraly, Eq. 0.71 models a composite material made a matrix reinforced with fibers. In the compound invariant $\mathfrak{I}_{\kappa}$, the unit vector $\mathbf{M}=\cos \theta \mathbf{G}_{1}+$ $\sin \theta \mathbf{G}_{2}$ defines the orientation along which the tissue is stiffest while $\kappa$ characterizes the degree of anisotropy, varying from 0 to 1 . When $\kappa=0$ it would model a composite with all the fibers perfectly aligned in the direction $\mathbf{M}$ and at $\kappa=1$ the fibers would be perfectly aligned in the perpendicular direction, $\mathbf{M}^{\perp}$. Finally, $\kappa=\frac{1}{2}$ models the case where fibers would have no preferential direction (isotropic). The parameters $\mu_{1}$ and $\mu_{2}$ are the effective stiffnesses of the matrix and fiber phases, respectively, both having dimensions of force per unit length. The parameter $\gamma$ is a non-dimensional parameter that governs the tissue's strain stiffening response.

The second Piola-Kirchhoff wall tension, $\mathbf{S}$, is written as

$$
\mathbf{S}=2 \frac{\partial w}{\partial \mathfrak{I}_{1}} \mathbf{1}+2 \frac{\partial w}{\partial \mathfrak{I}_{2}} \mathfrak{I}_{2} \mathbf{C}^{-1}+2 \frac{\partial w}{\partial \mathfrak{I}_{\kappa}}(\kappa \mathbf{1}+(1-2 \kappa) \mathbf{M} \otimes \mathbf{M})
$$

Substituting Eq. 0.71 into Eq. 0.72 one finds

$$
\mathbf{S}=\mu_{1}\left(\mathbf{1}-\mathbf{C}^{-1}\right)+\mu_{2} \mathrm{e}^{\gamma\left(\mathfrak{I}_{\kappa}-1\right)^{2}}\left(\mathfrak{I}_{\kappa}-1\right)(\kappa \mathbf{1}+(1-2 \kappa) \mathbf{M} \otimes \mathbf{M})
$$

noting that the second Piola-Kirchoff wall tension is related to the Cauchy wall tension via $\mathbf{t}=\frac{1}{\sqrt{\widetilde{I}_{2}}} \mathbf{F} \mathbf{S ~ F}^{\mathrm{T}}$.

The values of the model parameters $\mu_{1}, \mu_{2}, \gamma, \kappa$, and $\theta$ were determined by minimizing the sum of the squared difference between the stress computed from the inverse membrane analysis and those computed using Eq. 0.73. The nonlinear minimization was solved in Matlab (MathWorks, v. 7.14) where the model parameters were constrained such that: $\mu_{1}, \mu_{2}$, $\gamma>0,0 \leq \theta \leq \frac{\pi}{2}$, and $0 \leq \kappa \leq 1$. Due to the boundary effect in the stress analysis the perimeter ring of elements were excluded from the material parameter identification.

\subsection{Results}

Geometric Reconstruction A bulge inflation test to failure was performed on a ATAA collected from a male patient who was 55 years old. 


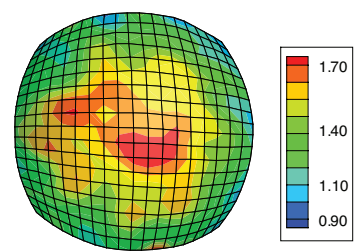

(a) Wall Tension

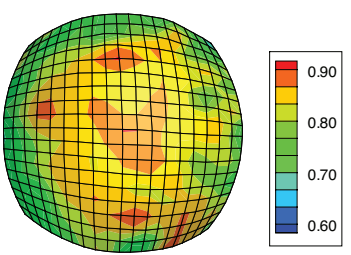

(b) Green-Lagrange strain

Figure 0.3: Contours of the magnitude of the (a) wall tension $(\mathrm{N} / \mathrm{m})$ and (b) Green-Lagrange strain at a pressure of $117 \mathrm{kPa}$. Adapted from [8].

The diameter of the aneurysm as determined by pre-surgical CT scan was $55 \mathrm{~mm}$. The mean thickness of the sample was $2.35 \mathrm{~mm}$. The pressure and DIC data during the bulge inflation tests were used to generate a deforming NURBS mesh and identify the local stress-strain response during the bulge inflation test. Using the pointwise stress-strain data, the spatial distribution of the mechanical properties was identified.

Using the experimental DIC point cloud a deforming NURBS mesh was generated of the ATAA sample.

Local Stress and Strain Response Figure 0.3 shows the distributions of the magnitude of the Cauchy wall tension, $\bar{t}$, and Green-Lagrangian strain, $\bar{E}$, at an applied pressure of $117 \mathrm{kPa}$ for a given ATAA sample. The distribution of wall tension and strain remained similar throughout the inflation of the specimen. In general, at each Gauss point both the normal strains and the planar shear strains were non-zero. To facilitate plotting of the local stress-strain response, the axes of principal strain were identified and the local stresses and strains were rotated into the principal strain axes. In [8], the three components of the wall tension in the principal strain axes, $\tilde{t}_{11}, \tilde{t}_{12}$, and $\tilde{t}_{22}$ were plotted against the principal stretches $\lambda_{1}$ and $\lambda_{2}$. As expected, the local stress-strain response showed the non-linear stiffening behaviour that is common in arteries. The shear stresses, $\tilde{t}_{12}$, were much smaller than the normal stresses, $\tilde{t}_{11}$ and $\tilde{t}_{22}$. In a small region where rupture eventually occurred, the ATAA appeared to yield. The locations of this localized yielding correspond to strain concentrations in zones where rupture initiates (Figure 0.3b).

Material Property Identification The proposed model for the elastic behaviour of the ATAA was able to fit the bulge inflation data well $(0.81<$ 


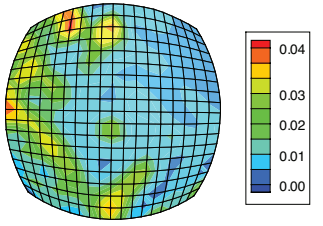

(a) $\mu_{1}(\mathrm{~N} / \mathrm{mm})$

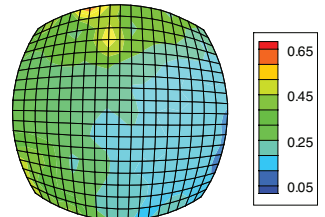

(b) $\mu_{2}(\mathrm{~N} / \mathrm{mm})$

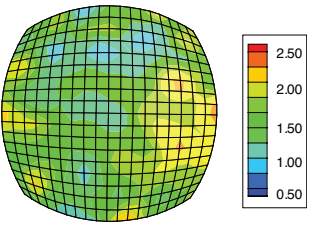

(c) $\gamma$

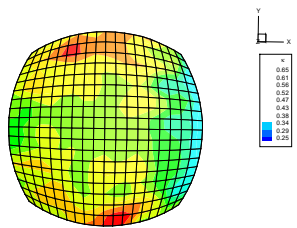

(d) $\kappa$

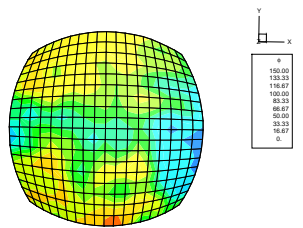

(e) $\theta(\mathrm{rad})$

Figure 0.4: Distribution of the identified material parameters over the ATAA. Adapted from [8].

$\left.\mathrm{R}^{2}<0.99\right)$. Lower values of the correlation coefficient were located in the small zone where rupture eventually occurred. Excluding this region the minimum value of $\mathrm{R}^{2}$ was 0.96 . The experimental data (points) and model fits (lines) for three Gauss points were shown in [8].

The distributions of the material parameters are plotted in Figure 0.4. Clearly the material parameters display a heterogeneous distribution. The parameter $\mu_{1}$ displayed the sharpest changes in value while the parameters $\mu_{2}, \kappa$, and $\gamma$ changed more gradually. Not surprisingly, the values of $\mu_{2}$ are an order of magnitude larger than $\mu_{1}$ reflecting the difference in stiffness between the collagen fibers and matrix. The values of $\kappa$ are approximately 0.5 in the center suggesting an isotropic organization of the collagen fibers. Towards the edges of the specimen the collagen fibers become more aligned signaling that the sample is regionally anisotropic. In Figure $0.4 \mathrm{e}$, the angle $\theta$ that defines the stiffest direction is plotted. Note that $\theta$ is defined locally relative to the horizontal meshlines. Keep in mind that when the value of $\kappa$ is approximately 0.5 , there is no stiffest direction.

This pointwise method was used to identify the distribution of material properties of 10 human ATAA samples [18]. Our method was able to capture the varying levels of heterogeneity in the ATAA from regional to local. The distributions of the material properties for each patient were examined to study the inter- and intra-patient variability. Future studies on 
the heterogeneous properties of the ATAA would benefit from some form of local structural analysis such as histology or multi-photon microscopy. The structural data and knowledge of the spatial trends should provide the information necessary to move from merely measuring the local material properties to uncovering the links that exist between the underlying microstructure and local properties.

\section{Characterization of hyperelastic material properties using a tension-inflation test and the virtual fields method}

In the previous section, it was shown that in some cases which are referred to as isostatic, it may be possible to derive the stress distribution independently of the material properties of the tissues. When strain distributions are also available, stress-strain curves can be derived locally and the inverse problem turns into a semi-forward problem [20], where the material parameters can be identified directly by fitting the curves with a model.

In case of hyperstatic situations, it is not possible to derive the stress distribution independently of the material properties of the tissues. A possible solution for the identification of local material properties may still be found using the Virtual Fields Method (VFM). The VFM is one of the techniques developed to identify the parameters governing constitutive equations, the experimental data processed for this purpose being displacement or strain fields. It will be shown in this chapter that one of its main advantages is the fact that, in several cases, the sought parameters can be directly found from the measurements, without resorting to a FE software.

The VFM relies on the Principle of Virtual Power (PVP) which is written with particular virtual fields.

\subsection{General Principle}

The PVP represents in fact the weak form the local equations of equilibrium which are classically introduced in mechanics of deformable media. Assuming a quasi-static transformation (absence of acceleration forces) and assuming the absence of body forces, the PVP can be written as follows for any domain defined by its volume $\omega(t)$ in the current configuration and by its external boundary $\partial \omega(t)$ :

$$
\underbrace{-\int_{\omega(t)} \boldsymbol{\sigma}:\left(\mathbf{G r a d} \mathbf{v}^{*}\right) d \omega}_{P_{i n t}^{*}}+\underbrace{\int_{\partial \omega(t)} \mathbf{t}_{\mathbf{n}} \cdot \mathbf{v}^{*} d s}_{P_{e x t}^{*}}=0
$$


where $\boldsymbol{\sigma}$ is the Cauchy stress tensor, $\mathbf{v}^{*}$ is a virtual velocity field defined across the volume of the solid, Grad $\mathbf{v}^{*}$ is the gradient of $\mathbf{v}^{*}, \mathbf{t}_{\mathbf{n}}$ are the tractions across the boundary (surface denoted $\partial \omega(t)), P_{i n t}^{*}$ is the virtual power of internal forces and $P_{e x t}^{*}$ is the virtual power of external forces.

A very important property is in fact that the equation above is satisfied for any kinematically admissible (KA) virtual field $\mathbf{v}^{\star}$. By definition, a KA virtual field must satisfy the boundary conditions of the actual velocity field in order to cancel the contribution of the resulting forces on the portion of the boundary along which actual displacement are prescribed. It must be pointed out that this requirement is not really necessary in all cases, but this point is not discussed here for the sake of simplicity. KA virtual fields are also assumed to be $\mathcal{C}_{0}$ functions [21].

\subsection{Example of application of the principle of virtual power for membranes}

The PVP may be a powerful tool to derive global or semi local equilibrium equations which eventually appear useful for the identification of material parameters. Here we illustrate this for deriving a useful equation for a hyperelastic membrane. This is purely for the sake of giving an example, but an infinity of other equations could be derived.

Let us consider a membrane-like structure made of a hyperelastic prestressed tissue. The membrane is defined by a 3D surface, namely defined by a set of points $\mathbf{M}\left(\xi_{1}, \xi_{2}\right)$, where $\left(\xi_{1}, \xi_{2}\right)$ are the surface parametric coordinates associated with the local basis $\left(\mathbf{g}^{1}, \mathbf{g}^{2}\right)$. Vector $\mathbf{g}^{1}$ points the direction of the maximum principal curvature and vector $\mathbf{g}^{2}$ points the direction of the minimum principal curvature. The thickness of the membrane is named $h\left(\xi_{1}, \xi_{2}\right)$ and we denote $\kappa^{1}\left(\xi_{1}, \xi_{2}\right)$ and $\kappa^{2}\left(\xi_{1}, \xi_{2}\right)$ respectively the maximum and minimum principal curvatures at $\left(\xi_{1}, \xi_{2}\right)$.

There is no particular assumption related to the thickness of the membrane but it is assumed that through-thickness shear is negligible. A third coordinate $\xi_{3}$ is introduced along the direction normal to the surface (throughthickness coordinate), with $\xi_{3}=0$ at the inner surface and $\xi_{3}=1$ at the outer surface.

Let us consider a quadrilateral patch across the membrane surface. This patch is denoted $n$ and we will apply the PVP on its volume. For that, the following virtual field $u^{*}$ is defined across the given patch $n$ :

$$
\mathbf{u}^{*}\left(\xi_{3}\right)=\frac{\left(1 / \kappa_{n}^{1}-h\right)\left(1 / \kappa_{n}^{2}-h\right)\left(1 / \kappa_{n}^{1}+1 / \kappa_{n}^{2}-2 h\right)}{\left(1 / \kappa_{n}^{1}-(1-\xi) h\right)\left(1 / \kappa_{n}^{2}-(1-\xi) h\right)} \mathbf{n}_{n}
$$


where $1 / \kappa_{n}^{1}$ is the average radius of curvature on the outer surface along the direction of the maximum principal curvature and $1 / \kappa_{n}^{2}$ is the average radius of curvature on the outer surface along the direction of the minimum principal curvature. The radii of curvature at any position $\xi_{3}$ between the inner $\left(\xi_{3}=0\right)$ and outer $\left(\xi_{3}=1\right)$ surfaces are then $\left(1 / \kappa_{n}^{1}-\left(1-\xi_{3}\right) h\right)$ and $\left(1 / \kappa_{n}^{2}-\left(1-\xi_{3}\right) h\right)$. Vector $\mathbf{n}_{n}$ points the direction normal to the surface.

The gradient of $\mathbf{u}^{*}$ may be written as follows:

$$
\begin{gathered}
\operatorname{Grad~u}^{*}\left(\xi_{3}\right)=\left[\frac{\left(1 / \kappa_{n}^{1}-h\right)\left(1 / \kappa_{n}^{2}-h\right)\left(1 / \kappa_{n}^{1}+1 / \kappa_{n}^{2}-2 h\right)}{\left(1 / \kappa_{n}^{1}-\left(1-\xi_{3}\right) h\right)^{2}\left(1 / \kappa_{n}^{2}-\left(1-\xi_{3}\right) h\right)}\right] \mathbf{g}_{n}^{1} \otimes \mathbf{g}_{n}^{1} \\
+\left[\frac{\left(1 / \kappa_{n}^{1}-h\right)\left(1 / \kappa_{n}^{2}-h\right)\left(1 / \kappa_{n}^{1}+1 / \kappa_{n}^{2}-2 h\right)}{\left(1 / \kappa_{n}^{1}-\left(1-\xi_{3}\right) h\right)\left(1 / \kappa_{n}^{2}-\left(1-\xi_{3}\right) h\right)^{2}}\right] \mathbf{g}_{n}^{2} \otimes \mathbf{g}_{n}^{2} \\
-\left[\frac{\left(1 / \kappa_{n}^{1}-h\right)\left(1 / \kappa_{n}^{2}-h\right)\left(1 / \kappa_{n}^{1}+1 / \kappa_{n}^{2}-2 h\right)}{\left(1 / \kappa_{n}^{1}-\left(1-\xi_{3}\right) h\right)^{2}\left(1 / \kappa_{n}^{2}-\left(1-\xi_{3}\right) h\right)}+\frac{\left(1 / \kappa_{n}^{1}-h\right)\left(1 / \kappa_{n}^{2}-h\right)\left(1 / \kappa_{n}^{1}+1 / \kappa_{n}^{2}-2 h\right)}{\left(1 / \kappa_{n}^{1}-\left(1-\xi_{3}\right) h\right)\left(1 / \kappa_{n}^{2}-\left(1-\xi_{3}\right) h\right)^{2}}\right] \mathbf{n}_{n} \otimes \mathbf{n}_{n}
\end{gathered}
$$

Plugging in and evaluating the integral expression for $P_{i n t}^{*}$ (cf. Eq.0.74):

$$
\begin{aligned}
P_{\text {int }}^{*}(t)= & -h(t)\left(1 / \kappa_{n}^{1}(t)-h(t)\right)\left(1 / \kappa_{n}^{2}(t)-h(t)\right)\left(1 / \kappa_{n}^{1}(t)+1 / \kappa_{n}^{2}(t)-2 h(t)\right) \\
& \int_{0}^{1}\left[\frac{\sigma_{11, n}^{w}\left(t, \xi_{3}\right)-\sigma_{33, n}^{w}\left(t, \xi_{3}\right)}{\left(1 / \kappa_{n}^{1}-\left(1-\xi_{3}\right) h\right)^{2}\left(1 / \kappa_{n}^{2}-\left(1-\xi_{3}\right) h\right)}\right. \\
& \left.+\frac{\sigma_{22, n}^{w}\left(t, \xi_{3}\right)-\sigma_{33, n}^{w}\left(t, \xi_{3}\right)}{\left(1 / \kappa_{n}^{1}-\left(1-\xi_{3}\right) h\right)\left(1 / \kappa_{n}^{2}-\left(1-\xi_{3}\right) h\right)^{2}}\right] A_{n}\left(t, \xi_{3}\right) \mathrm{d} \xi_{3} \quad(0.77)
\end{aligned}
$$

where $A_{n}\left(t, \xi_{3}\right)$ is the area of patch $n$ at radial position $\xi_{3}$ and may be written:

$$
A_{n}\left(t, \xi_{3}\right)=\left(1 / \kappa_{n}^{1}(t)-\left(1-\xi_{3}\right) h(t)\right)\left(1 / \kappa_{n}^{2}(t)-\left(1-\xi_{3}\right) h(t)\right) \Theta_{n}^{1}(t) \Theta_{n}^{2}(t)
$$

where $\Theta_{n}^{1}$ and $\Theta_{n}^{2}$ are two angles defining the angular sector of patch $n$ along the directions of the maximum and minimum principal curvatures, respectively. Introducing the expression of $A_{n}\left(t, \xi_{3}\right)$ into Eq.0.77, we obtain:

$$
\begin{gathered}
P_{i n t}^{*}(t)=-h(t)\left(1 / \kappa_{n}^{1}(t)-h(t)\right)\left(1 / \kappa_{n}^{2}(t)-h(t)\right)\left(1 / \kappa_{n}^{1}(t)+1 / \kappa_{n}^{2}(t)-2 h(t)\right) \\
\Theta_{n}^{1}(t) \Theta_{n}^{2}(t) \int_{0}^{1}\left[\frac{\sigma_{11, n}^{w}\left(t, \xi_{3}\right)-\sigma_{33, n}^{w}\left(t, \xi_{3}\right)}{\left(1 / \kappa_{n}^{1}-\left(1-\xi_{3}\right) h\right)}+\frac{\sigma_{22, n}^{w}\left(t, \xi_{3}\right)-\sigma_{33, n}^{w}\left(t, \xi_{3}\right)}{\left(1 / \kappa_{n}^{2}-\left(1-\xi_{3}\right) h\right)}\right] \mathrm{d} \xi_{3}
\end{gathered}
$$


Regarding the virtual work on the boundaries, shear stresses are null so only the virtual work of the internal pressure needs to be considered:

$$
\begin{array}{r}
P_{\text {ext }}^{*}(t)=P(t)\left(1 / \kappa_{n}^{1}(t)-h(t)\right)\left(1 / \kappa_{n}^{2}(t)-h(t)\right) \Theta_{n}^{1}(t) \Theta_{n}^{2}(t) \\
\left(1 / \kappa_{n}^{1}(t)+1 / \kappa_{n}^{2}(t)-2 h(t)\right)
\end{array}
$$

so combining all the equations we have:

$$
P(t)=h(t) \int_{0}^{1}\left[\frac{\sigma_{11, n}^{w}\left(t, \xi_{3}\right)-\sigma_{33, n}^{w}\left(t, \xi_{3}\right)}{\left(1 / \kappa_{n}^{1}-\left(1-\xi_{3}\right) h\right)}+\frac{\sigma_{22, n}^{w}\left(t, \xi_{3}\right)-\sigma_{33, n}^{w}\left(t, \xi_{3}\right)}{\left(1 / \kappa_{n}^{2}-\left(1-\xi_{3}\right) h\right)}\right] \mathrm{d} \xi_{3}
$$

Finally the obtained equation is a generalized expression of the traditional Laplace law commonly used in biomechanics of soft tissues [22].

\subsection{Identification of hyperelastic parameters using the VFM}

The principle of virtual power (PVP) has been used for the identification of material properties since 1990 through the virtual fields method (VFM), which is an inverse method based on the use of full-field deformation data $[23,24,21]$. The VFM was recently applied to the identification of uniform material properties in arterial walls [24].

The first step of the VFM consists in introducing the constitutive equations. In the case of hyperelasticity, Equation 0.74 becomes:

$$
-\int_{\omega(t)}\left(J^{-1} \mathbf{F} \frac{\partial \psi}{\partial \mathbf{E}} \mathbf{F}^{T}\right):\left(\mathbf{G r a d}^{*}\right) d \omega+\int_{\partial \omega(t)} \mathbf{t}_{\mathbf{n}} \cdot \mathbf{v}^{*} d s=0
$$

This equation being satisfied for any KA virtual field, any new KA virtual field provides a new equation. The VFM relies on this property by writing Eq. 0.82 above with a set of KA virtual fields chosen a priori [25]. The number of virtual fields and their type depend on the nature of the strain energy function. Two different cases can be distinguished.

- Case \#1: the strain energy density function depends linearly on the sought parameters. Writing Eq. 0.82 with as many virtual fields as unknowns leads to a system of linear equations which provides the sought parameters after inversion.

- Case \#2: the strain energy density function involve nonlinear relations with respect to the constitutive parameters. In this case, identification must be performed by minimizing a cost-function derived from Eq. 0.82. 
Let us illustrate this with the strain energy function of Eq. 0.71. It provides a membrane constitutive equation, i.e. it yields the tension and not the Cauchy stress so the integrals will be written across a given surface $\nu(t)$ figuring a portion of the membrane:

$$
\begin{aligned}
&-\mu_{1} \int_{\nu(t)} \frac{1}{\sqrt{\mathfrak{I}_{2}}}\left(\mathbf{B}-\sqrt{\mathfrak{I}_{2}} \mathbf{1}\right):\left(\mathbf{G r a d ~}^{*}\right) d \nu \\
&-\mu_{2} \kappa \int_{\nu(t)} \frac{1}{\sqrt{\mathfrak{I}_{2}}} \mathrm{e}^{\gamma\left(\mathfrak{I}_{\kappa}-1\right)^{2}}\left(\mathfrak{I}_{\kappa}-1\right) \mathbf{B}:\left(\mathbf{G r a d}^{*}\right) d \nu \\
&-\mu_{2}(1-2 \kappa) \int_{\nu(t)} \frac{1}{\sqrt{\mathfrak{I}_{2}}} \mathrm{e}^{\gamma\left(\mathfrak{I}_{\kappa}-1\right)^{2}}\left(\mathfrak{I}_{\kappa}-1\right)\left(\mathbf{F M} \otimes \mathbf{M F}^{T}\right):\left(\mathbf{G r a d} \mathbf{v}^{*}\right) d \nu \\
& \quad+\int_{\nu(t)} \mathbf{t}_{\mathbf{n}} \cdot \mathbf{v}^{*} d l=0
\end{aligned}
$$

The equation may be rewritten such as:

$$
\mu_{1} A_{i j}+\mu_{2} \kappa B_{i j}(\gamma)+\mu_{2}(1-2 \kappa) C_{i j}(\gamma, \theta)=L_{i j}
$$

where $A_{i j}, B_{i j}, C_{i j}$ and $L_{i j}$ can be evaluated directly from the experimental measurements. Index $i$ is for different possible choices of virtual fields and index $j$ is for different possible stages of the experiment for which deformations and loads are measured.

Eq. 0.84 is an equation of the unknown material parameters for each choice of virtual field $i$ and at every stage $j$ of the test. The equation is linear in $\mu_{1}, \mu_{2} \kappa$ and $\mu_{2}(1-2 \kappa)$ but it is nonlinear in $\gamma$ and $\theta$. The solution is found by minimizing a cost function defined such as:

$$
\sum_{i} \sum_{j}\left(\mu_{1} A_{i j}+\mu_{2} \kappa B_{i j}(\gamma)+\mu_{2}(1-2 \kappa) C_{i j}(\gamma, \theta)-L_{i j}\right)^{2}
$$

This cost function can be minimized by the simplex method or using a genetic algorithm in case of multiple minima. The chosen virtual fields and other details about the experiments can be found in $[24,26]$ for applications to blood vessels.

A recent extension of the method was proposed for the inverse characterization of regional, nonlinear, anisotropic properties of the murine aorta [27]. Full-field biaxial data were collected using a panoramic-digital image correlation system and the VFM was used to estimate values of material parameters regionally for a microstructurally motivated constitutive relation. 
The experimental-computational approach was validated by comparing results to those from standard biaxial testing. Results for the non-diseased suprarenal abdominal aorta from apolipoprotein-E null mice revealed material heterogeneities, with significant differences between dorsal and ventral as well as between proximal and distal locations, which may arise in part due to differential perivascular support and localized branches. Overall results were validated for both a membrane and a thick-wall model that delineated medial and adventitial properties.

Whereas full-field characterization can be useful in the study of normal arteries, we submit that it will be particularly useful for studying complex lesions such as aneurysms. Indeed, many vascular disorders, including aortic aneurysms and dissections, are characterized by localized changes in wall composition and structure. Notwithstanding the importance of histopathologic changes that occur at the microstructural level, macroscopic manifestations ultimately dictate the mechanical functionality and structural integrity of the aortic wall. Understanding structure-function relationships locally is thus critical for gaining increased insight into conditions that render a tissue susceptible to disease or failure.

\section{Conclusion}

In this chapter, after a brief review of the constitutive relations commonly used for soft tissues, two recent developments of the author's experience were presented to illustrate the potential of digital image correlation and inverse methods in experimental biomechanics of soft tissues.

The inverse problems, including the semi-forward problems [20], posed by the identification of material properties in soft biological tissues are not the simplest due to the complex microstructure of soft biological tissues, their finite range of deformation, their inter-individual variability, their anisotropy, their point-dependent non-linear behaviour, and their permanent functional adaptation to the environment. Determining the mechanical properties of such tissues has nevertheless become a field of intense research since stress analysis in the tissues has been shown to be meaningful for medical diagnosis in a number of medical applications as for instance in the context of vascular medicine, indicating the risk of rupture of an aneurysm [28] or the risk of stroke [29].

The current chapter has focused on in vitro characterization. The in vivo identification of soft tissues present other important issues. They suppose both the existence of reliable experimental facilities for inducing a mechanical stimulus (natural blood pressure variations, local external compression, shear waves [30]) and the existence of imaging devices for measuring the 
response of tissues (Ultrasound Imaging [31], Magnetic Resonance Imaging [32] or Optical Coherence Tomography [33]). In all these situations where some elements of the response of soft tissues subjected to mechanical stimuli are measured, the access to the mechanical parameters is never direct and inverse problems have to be posed and solved. The inverse problems posed by the in vivo identification of soft tissues will be discussed more specifically in chapter 5 and chapter 6 of this book.

\section{Acknowledgements}

The author of this chapter would like to thank all the students and colleagues who participated to cited studies: Aaron Romo, Pierre Badel, Frances Davies, Ambroise Duprey, Jean-Pierre Favre, Olfa Trabelsi, Yuanming Luo, Jia Lu, Matt Bersi, Chiara Bellini, Katia Genovese, Jay Humphrey. 


\section{List of references}

[1] Millard F Beatty. Topics in finite elasticity: hyperelasticity of rubber, elastomers, and biological tissues. Applied Mechanics Reviews, 40(12):1699-1734, 1987.

[2] KY Volokh. Modeling failure of soft anisotropic materials with application to arteries. Journal of the Mechanical Behaviour of Biomedical Materials, 4(8):1582-1594, 2011.

[3] Victor H Barocas and Robert T Tranquillo. An anisotropic biphasic theory of tissue-equivalent mechanics: the interplay among cell traction, fibrillar network deformation, fibril alignment, and cell contact guidance. Journal of biomechanical engineering, 119(2):137-145, 1997.

[4] Aydin Tözeren and Richard Skalak. Interaction of stress and growth in a fibrous tissue. Journal of theoretical biology, 130(3):337-350, 1988.

[5] JD Humphrey and KR Rajagopal. A constrained mixture model for growth and remodeling of soft tissues. Mathematical models and methods in applied sciences, 12(03):407-430, 2002.

[6] S Baek, KR Rajagopal, and JD Humphrey. A theoretical model of enlarging intracranial fusiform aneurysms. Journal of biomechanical engineering, 128(1):142-149, 2006.

[7] Jay D Humphrey. Cardiovascular solid mechanics: cells, tissues, and organs. Springer Science \& Business Media, 2013.

[8] Frances M Davis, Yuanming Luo, Stéphane Avril, Ambroise Duprey, and Jia Lu. Pointwise characterization of the elastic properties of planar soft tissues: application to ascending thoracic aneurysms. Biomechanics and modeling in mechanobiology, 14(5):967-978, 2015.

[9] T Belytschko, W K Liu, D Organ, M Fleming, and P Krysl. Meshless methods: an overview and recent developments. Computer Methods in Applied Mechanics and Engineering, 139:3-47, 1996.

[10] Jia Lu. Isogeometric contact analysis: Geometric basis and formulation for frictionless contact. Computer Methods in Applied Mechanics and Engineering, 200(5-8):726-741, January 2011.

[11] Albert Edward Green and John Edward Adkins. Large elastic deformations. Clarendon Press Oxford, 1970.

[12] J Lu, X L Zhou, and M L Raghavan. Computational Method for Inverse Elastostatics for Anisotropic Hyperelastic Solids. International Journal for Numerical Methods in Engineering, 69:1239-1261, 2007.

[13] Jia Lu, Xianlian Zhou, and Madhavan L Raghavan. Inverse method of stress analysis for cerebral aneurysms. Biomechanics and modeling in mechanobiology, 7(6):477-86, December 2008.

[14] Xuefeng Zhao, Madhavan L Raghavan, and Jia Lu. Identifying heterogeneous anisotropic properties in cerebral aneurysms: a pointwise 
approach. Biomechanics and modeling in mechanobiology, 10(2):177189, 2011.

[15] Karol Miller and Jia Lu. On the prospect of patient-specific biomechanics without patient-specific properties of tissues. Journal of the mechanical behavior of biomedical materials, 27:154-166, 2013.

[16] Jia Lu, Shouhua Hu, and Madhavan L Raghavan. A shell-based inverse approach of stress analysis in intracranial aneurysms. Annals of biomedical engineering, 41(7):1505-1515, 2013.

[17] Katia Genovese, Luciana Casaletto, Jay D Humphrey, and Jia Lu. Digital image correlation-based point-wise inverse characterization of heterogeneous material properties of gallbladder in vitro. Proceedings of Royal Society A, 470(2167), 2014.

[18] Frances M Davis, Yuanming Luo, Stéphane Avril, Ambroise Duprey, and Jia Lu. Local mechanical properties of human ascending thoracic aneurysms. Journal of the Mechanical Behaviour of Biomedical Materials, 2016. Accepted.

[19] T Christian Gasser, Ray W Ogden, and Gerhard A Holzapfel. Hyperelastic modelling of arterial layers with distributed collagen fibre orientations. Journal of the royal society interface, 3(6):15-35, 2006.

[20] Claire Morin and Stéphane Avril. Inverse problems in the mechanical characterization of elastic arteries. In MRS Bulletin, volume 40. Materials Research Society, 2015.

[21] Fabrice Pierron and Michel Grédiac. The virtual fields method: extracting constitutive mechanical parameters from full-field deformation measurements. Springer Science \& Business Media, 2012.

[22] Yuan-cheng Fung. Biomechanics: mechanical properties of living tissues. Springer Science \& Business Media, 2013.

[23] Stéphane Avril, Marc Bonnet, Anne-Sophie Bretelle, Michel Grediac, François Hild, Patrick Ienny, Felix Latourte, Didier Lemosse, Stéphane Pagano, Emmanuel Pagnacco, et al. Overview of identification methods of mechanical parameters based on full-field measurements. Experimental Mechanics, 48(4):381-402, 2008.

[24] Stéphane Avril, Pierre Badel, and Ambroise Duprey. Anisotropic and hyperelastic identification of in vitro human arteries from full-field optical measurements. Journal of Biomechanics, 43(15):2978-2985, 2010.

[25] Michel Grédiac. Principe des travaux virtuels et identification. Comptes Rendus de l'Académie des Sciences, pages 1-5, 1989. In French with abridged English version.

[26] Jin-Huan Kim, Stéphane Avril, Ambroise Duprey, and Jean-Pierre Favre. Experimental characterization of rupture in human aortic aneurysms using full-field measurement technique. Biomechanics and Modeling in Mechanobiology, 11(6):841-854, 2012. 
[27] Matthew R Bersi, Chiara Bellini, Paolo Di Achille, Jay D Humphrey, Katia Genovese, and Stéphane Avril. Novel methodology for characterizing regional variations in material properties of murine aortas. Journal of Biomechanical Engineering, 2016. In press.

[28] Mark F Fillinger, Steven P Marra, ML Raghavan, and Francis E Kennedy. Prediction of rupture risk in abdominal aortic aneurysm during observation: wall stress versus diameter. Journal of vascular surgery, 37(4):724-732, 2003.

[29] Zhi-Yong Li, Simon PS Howarth, Tjun Tang, and Jonathan H Gillard. How critical is fibrous cap thickness to carotid plaque stability? a flow-plaque interaction model. Stroke, 37(5):1195-1199, 2006.

[30] Fanny Frauziols, Jérôme Molimard, Laurent Navarro, Pierre Badel, Magalie Viallon, Rodolphe Testa, and Stéphane Avril. Prediction of the biomechanical effects of compression therapy by finite element modeling and ultrasound elastography. IEEE Transactions on Biomedical Engineering, 62(4):1011-1019, 2015.

[31] J. Bercoff, M. Tanter, and M. Fink. Supersonic shear imaging: a new technique for soft tissue elasticity mapping. IEEE Transactions on Ultrasonics, Ferroelectrics and Frequency Control, 51(4):396 -409, April 2004.

[32] Sabine F Bensamoun, Stacie I Ringleb, Laurel Littrell, Qingshan Chen, Michael Brennan, Richard L Ehman, and Kai-Nan An. Determination of thigh muscle stiffness using magnetic resonance elastography. Journal of Magnetic Resonance Imaging, 23(2):242-247, February 2006.

[33] Hiroshi Yabushita, Brett E Bouma, Stuart L Houser, H Thomas Aretz, Ik-Kyung Jang, Kelly H Schlendorf, Christopher R Kauffman, Milen Shishkov, Dong-Heon Kang, Elkan F Halpern, et al. Characterization of human atherosclerosis by optical coherence tomography. Circulation, 106(13):1640-1645, 2002. 